\title{
PRELIMINARY DESIGN OF AN AUTOMATED RAPID MAXILLARY EXPANDER FOR DENTO-FACIAL APPLICATION
}

\author{
A. A. Sharizli ${ }^{1}$, F.A. Zabir ${ }^{1}$, N.A. Abu Osman ${ }^{1}$, Z. Radzi $^{1}$, A.A.Shaifizul ${ }^{2}$ \\ ${ }^{1}$ Department of Biomedical Engineering, University of Malaya, Kuala Lumpur, Malaysia \\ ${ }^{2}$ Department of Mechanical Engineering, University of Malaya, Kuala Lumpur, Malaysia \\ email : azuan@um.edu.my, fauroraz@gmail.com
}

\section{INTRODUCTION}

The objective of maxillary expansion is to correct maxillo mandibular transverse discrepancies by skeletal (orthopedic) expansion of the maxilla while avoiding any dental side effects. [1]. For a rapid maxillary expansion (RME) device, the expansion of maxilla is accomplished through heavy forces that are capable of separating the midpalatal suture at the rate of 0.2 to $0.5 \mathrm{~mm}$ per day[1]. This procedure can be done rapidly or slowly depending upon the patient's age and treatment plan [2]. In this technique, the orthodontist will custom fit an appliance on the upper molars of a patient (Figure 1). Either the patient or the parent will turn the appliance every day throughout a prescribed period. The current expanders are turned by first placing a pin in a tiny hole (capstan). The inserted pin will then push the hole to the rear of the expander. Naturally, the patient is asked to turn the active part of the screw to a certain counts.

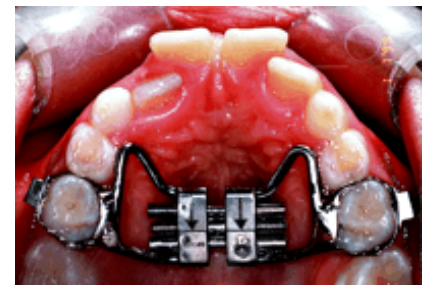

Figure 1: Butterfly RME fixed to the upper molars.

The load of work involved in the treatment is considered inefficient since they require numerous clinical visits in order to manipulate the components or sometimes to replace the jackscrews. Based on the issue that patients need to activate the device manually, keep track of the number of activations with no knowledge of magnitude of force applied, an automated RME represents a reasonable alternative to using conventional maxillary expansion appliances.

\section{METHODS}

To overcome the limitations of conventional expansion appliances, the automated RME appliance was designed and developed using a combination of butterfly expander (Figure 1), a micro gear, a nano motor and also a microcontroller to produce low and continuous pressure on the midpalatal suture. The first phase is a preliminary design of an automated RME system, which eliminates the usage of a pin to move the expander. The second phase is mechanical modifications of the butterfly expander. By replacing the tiny hole section of the screw with a micro gear, this will provide a direct interface to the micro motor. As the micro motor spins, this will effectively turn the micro gear to a desired position. The third phase is a microcontroller system design. Microcontroller circuit drawing utilizes PCB circuit design. The next phase is system integration. In this phase, all the subsystems inclusive of the microcontroller system, the nano motor and a modified butterfly expander will be integrated (Figure 2). The fifth phase is testing and running clinical trials. In this phase, testing of the new automated RME appliance will consist of evaluating the rapidity of dental arch movement, measuring the forces applied to the RME and executing preliminary clinical trials.

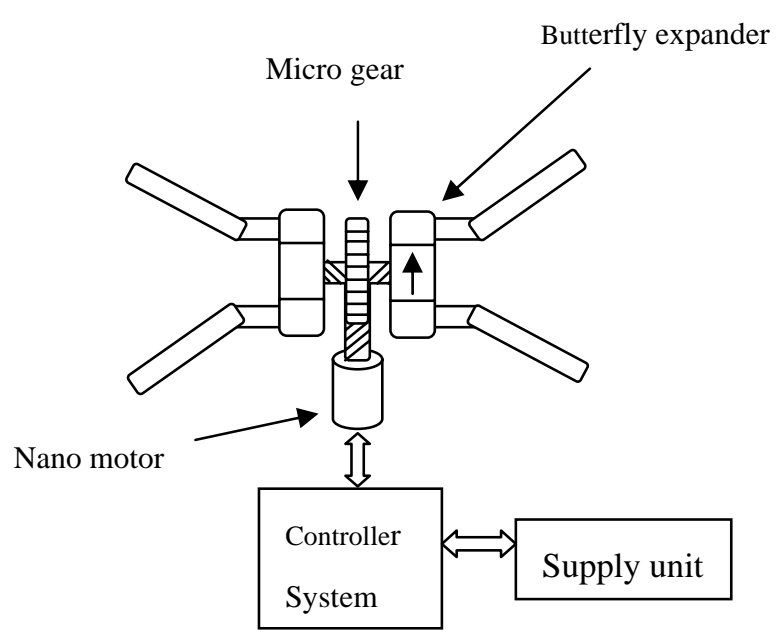

Figure 2: System integration of an automated RME

\section{RESULTS AND DISCUSSION}

At present, we are in the process of assembling the subparts and making sure that the parts collaborate perfectly to validate our structural design. Future plans include a structural analysis using the finite element method softwares (i.e ProE or Abaqus) to analyze the data. The experimental results will then be used to assist in improving the design of the system.

\section{CONCLUSIONS}

The newly developed automated RME appears to have a number of advantages over the traditional appliance. For a start, it is highly convenient for patient undergoing RME treatment. The construction of the device is now in the system integration phase. The next phase in progress is to determine the feasibility of the design and to assess the fundamental performance whether or not it fulfills the design expectations.

\section{REFERENCES}

1. Haas AJ. Angle Orthod, 31, 73-90, 1961.

2. Cross DL et al.. European Journal of Orthod , 22, 519-528, 2000. 